\title{
P30 Interplay of Genetic and Environmental Factors Determining Carotid and Femoral Intima-media Thickness and Plaques
}

Adam Domonkos Tarnoki*, Rebeka Debreceni, Greta Janositz, Kata Gyulay, Jokkel Zsofia, Viktor Berczi, Helga Szabo, Anita Hernyes, David Laszlo Tarnoki

Department of Radiology, Semmelweis University, Budapest, Hungary

\section{ABSTRACT}

Introduction: Intima-media thickness (IMT) of carotid and femoral artery and plaque formation are both markers of subclinical cardiovascular risk, despite these two process are not simultaneous. Our aim was to investigate their genetic and environmental background.

Methods: 54 monozygotic (MZ) and 32 dizygotic (DZ) healthy, adult (mean age: $54.3 \pm 13.8$ years) Hungarian twins underwent B-mode ultrasound of bilateral common (CCA), internal carotid (ICA) and common femoral arteries (CFA) with automatic IMT measurement and plaque analysis (Samsung Prestige RS80).

Results: In our healthy sample, most of the plaques were located on the carotid arteries (68\%), while the prevalence was $33 \%$ on the femoral arteries. The mean IMT of CCA and CFA was significantly increased in the presence of plaques $(p<0.05)$, while IMT of ICA was not affected by the presence of plaques. Increased IMT was in a close relation with the presence of femoral plaques. However, there was no significant correlation between IMT and the number or volume of plaques. Segment-specific heritability of IMT changed between 0.20 and 0.93 . The prevalence of soft plaques was the highest (59\%) on carotid arteries, while the majority of plaques $(42 \%)$ was mixed on the femoral arteries. Increased values of IMT were in correlation with the presence of calcified and mixed carotid, and calcified femoral plaques $(p<0.05)$, while there was no correlation with the occurrence of soft plaques.

Conclusion: Intima-media thickening and plaque formation has a different background, depending on the individual genetic predisposition. In addition, treatment efficacy of increased IMT may vary segment-specifically.

(c) 2019 Association for Research into Arterial Structure and Physiology. Publishing services by Atlantis Press International B.V. This is an open access article distributed under the CC BY-NC 4.0 license (http://creativecommons.org/licenses/by-nc/4.0/) 